\title{
Safety Evaluation of Bifidobacterium breve IDCC4401 Isolated from Infant Feces for Use as a Commercial Probiotic
}

\author{
In Young Choi ${ }^{1,2 \dagger}$, Jinhee Kim ${ }^{3 \dagger}$, Su-Hyeon Kim ${ }^{1,2}$, O-Hyun Ban ${ }^{4}$, Jungwoo Yang ${ }^{4}$, and \\ Mi-Kyung Park ${ }^{1,2 *}$ \\ ${ }^{1}$ School of Food Science and Biotechnology, Kyungpook National University, Daegu 41566, Republic of Korea \\ ${ }^{2}$ Food and Bio-Industry Research Institute, Kyungpook National University, Daegu 41566, Republic of Korea \\ ${ }^{3}$ Drug Information Platform Center, Korea Research Institute of Chemical Technology, Daejeon 34114, Republic of Korea \\ ${ }^{4}$ Ildong BioScience, Pyeongtaek 17957, Republic of Korea
}

Previously, our research group isolated Bifidobacterium breve IDCC4401 from infant feces as a potential probiotic. For this study, we evaluated the safety of $B$. breve IDCC4401 using genomic and phenotypic analyses. Whole genome sequencing was performed to identify genomic characteristics and investigate the potential presence of genes encoding virulence, antibiotic resistance, and mobile genetic elements. Phenotypic analyses including antibiotic susceptibility, enzyme activity, production of biogenic amines (BAs), and proportion of D-/L-lactate were evaluated using E-test, API ZYM test, high-performance liquid chromatography (HPLC), and D-/L-lactic acid assay respectively. The genome of $B$. breve IDCC4401 consists of 2,426,499 bp with a GC content of $58.70 \%$ and 2,016 coding regions. Confirmation of the genome as $B$. breve was provided by its $98.93 \%$ similarity with $B$. breve DSM20213. Furthermore, B. breve IDCC4401 genes encoding virulence and antibiotic resistance were not identified. Although $B$. breve IDCC4401 showed antibiotic resistance against vancomycin, we confirmed that this was an intrinsic feature since the antibiotic resistance gene was not present. B. breve IDCC4401 showed leucine arylamidase, cystine arylamidase, a-galactosidase, $\beta$-galactosidase, and $\alpha$-glucosidase activities, whereas it did not show production of harmful enzymes such as $\beta$-glucosidase and $\beta$-glucuronidase. In addition, $B$. breve IDCC4401 did not produce any tyramine, histamine, putrescine, cadaverine, or 2-phenethylamine, which are frequently detected BAs during fermentation. B. breve IDCC4401 produced $95.08 \%$ of L-lactate and $4.92 \%$ of Dlactate. Therefore, our findings demonstrate the safety of B. breve IDCC 4401 as a potential probiotic for use in the food industry.

Keywords: Safety evaluation, Bifidobacterium breve, probiotics

Received: March 25, 2021
Accepted:May 21,2021
First published online:
May 24, 2021
*Corresponding author
Phone: +82-53-950-5776
Fax: +82-53-950-6772
E-mail: parkmik@knu.ac.kr
'I.Y. Choi and J. Kim contributed
equally to this work.
pISSN 1017-7825
elSSN 1738-8872
Copyright@ 2021 by
The Korean Society for
Microbiology and
Biotechnology

\section{Introduction}

The United Nations and World Health Organization (WHO) [1] define probiotics as "live microorganisms which confer health benefits on the host when administered in adequate amounts". Probiotics provide their major health benefits by inhibiting the growth of pathogens in the gastrointestinal tract, reducing the risk of colon cancer and bowel disease, controlling serum cholesterol levels, facilitating digestion, and improving nutrient absorption [2-5]. In addition, they contribute to a balanced gut microbial community and strengthen the immune system. Due to these health benefits and advantages, customer interest in probiotic foods has been increasing. Probiotics have been applied in a wide range of industries such as food, alcoholic beverage, periodontal disease treatment, animal feed and cosmetics $[4,6]$. As a result, the global probiotic market size was estimated at approximately 48 billion USD in 2018 and is predicted to reach 77.09 billion USD with a compound annual growth rate of $6.9 \%$ by 2025 [7].

Most probiotics are gram-positive, catalase-negative, and non-pathogenic bacteria. There are several bacteria that are used as probiotics such as Lactobacillus spp., Bifidobacterium spp., Propionibacterium spp., Peptostreptococcus productus, Bacillus spp., Lactococcus spp., Enterococcus faecium, Pediococcus spp., and Streptococcus spp.. Of these, Lactobacillius spp. and Bifidobacterium spp. were the most commonly used as probiotics in the food and pharmaceutical industries [4, 8]. Bifidobacterium spp. were first isolated from the feces of breast-fed infants in 1899 [9] and are among the most dominant probiotics during the neonatal period, especially in breast-fed infants [10]. Previous studies have suggested that Bifidobacterium spp. are predominantly found in the feces of healthy breast-fed infants contributing to decreased infant diarrhea [11] as well as providing various health benefits as a 
probiotic [3, 12]. In particular, Bifidobacterium spp. play an important role in balancing the gut microbiota such that the food and pharmaceutical industries use Bifidobacterium spp. as a starter culture in their products [13]. Therefore, searching new lactic acid bacteria for commercial application is required.

Recently, our research group isolated B. breve IDCC4401 from infant feces in Korea. Although Lactobacillus and Lactococcus are considered as "generally recognized as safe (GRAS)," Bifidobacterium spp. require safety evaluation [14]. For example, some probiotics possess genes encoding virulence, antibiotic resistance, and mobile genetic elements, and have deleterious metabolic activities [production of D-lactate, enzyme activity, and biogenic amines (BAs)] [4, 10, 15-17]. Thus, an FAO/WHO Expert Consultation has recognized and emphasized the necessity for systematic evaluation guidelines for probiotics prior to their commercialization [18]. Following these guidelines for probiotics in food, the safety of isolated novel strain of B. breve IDCC4401 was evaluated through genomic analysis to determine the presence of virulence genes, antibiotic resistance genes, and mobile genetic elements. In addition, B. breve IDCC4401 was examined for antibiotic susceptibility, enzyme activity, production of BAs, and the proportion of D-/L-lactate formed during incubation.

\section{Materials and Methods}

\section{Bacterial Culture and Growth Conditions}

B. breve IDCC4401 obtained from Ildong BioScience Co. (Korea) and grown in $15 \mathrm{ml}$ of MRS broth (Difco Laboratories Inc., USA) at $37^{\circ} \mathrm{C}$ for $24 \mathrm{~h}$ with $0.5 \% \mathrm{CO}_{2}$ in a static incubator. After centrifugation at $6,000 \times g$ for 15 min, the pellet was resuspended in PBS (pH 7.4; Life Technologies Ltd., UK) and its concentration was adjusted to $10^{8} \mathrm{CFU} / \mathrm{ml}$ and $10^{9} \mathrm{CFU} / \mathrm{ml}$ by measuring the optical density at $640 \mathrm{~nm}$.

\section{Whole Genome Sequencing of B. breve IDCC4401}

Genomic DNA of B. breve IDCC4401 was extracted using a Maxwell 16 LEV Blood DNA Kit and a Maxwell 16 Buccal Swab LEV DNA Purification Kit (Promega Co., USA) according to the manufacturer's instructions. Genome sequencing was performed by Macrogen Inc. (Korea) using a PacBio RS II instrument (Pacific Biosciences of California Inc., USA) with an Illumina platform (Illumina Inc.,USA). Raw data were assembled using the Hierarchical Genome Assembly Process and the assembled gene was predicted and annotated using Prokka v1.13. For additional annotation, predicted protein sets were then analyzed using InterProScan v5.30-69.0 and psiblast v2.4.0 with EggNOG DB v4.5. Average nucleotide identity (ANI) was calculated using average nucleotide identity.py script of PYANI v0.2.10 with ANIm option. To confirm the presence of antibiotic resistance genes and virulence factors, all identified coding sequences (CDS) were compared against the Comprehensive Antibiotic Resistance Database (CARD) [19] and Virulence Factor Database (VFDB) [20], respectively. Prophage regions were identified using the PHASTER web-based program [21]. Transposases were annotated using BLASTP and transposases and conjugal transfer proteins were retrieved from the NCBI GenBank. Circular maps depicting each contig were generated using Circos v0.69.3. The complete genome sequence of B. breve IDCC4401 was made available in the GenBank database under nucleotide sequence accession number KP325411.

\section{Antibiotic Susceptibility of $B$. breve IDCC4401}

The antibiotic susceptibility of $B$. breve IDCC 4401 was determined by the E-test method against 9 antibiotics according to the European Food Safety Authority (EFSA) guidelines [17]. An overnight culture of bacteria $\left(10^{8} \mathrm{CFU} / \mathrm{ml}\right)$ was swabbed onto $15-\mathrm{cm}$ diameter MRS agar plates with a sterilized cotton swab prior to placing an E-test strip (Liofilchem Inc., Italy) on the surface of the plate. After incubation at $37^{\circ} \mathrm{C}$ for $18 \mathrm{~h}$, the relevant inhibition ellipse intersected the strip and the minimum inhibitory concentration was determined at complete inhibition. Finally, antibiotic susceptibility of B. breve IDCC4401 was determined following the guidelines of the EFSA [17].

\section{Enzyme Activities of B. breve IDCC4401}

The enzyme activities of $B$. breve IDCC4401 were determined using the API ZYM Kit (Biomerieux Inc., France) according to the manufacturer's instructions. The overnight culture of $B$. breve IDCC4401 $\left(10^{9} \mathrm{CFU} / \mathrm{ml}\right)$ was added into a cupule containing different substrate solutions and incubated at $37^{\circ} \mathrm{C}$ for $4 \mathrm{~h}$. One drop of ZYM A and ZYM B reagents was added sequentially prior to incubation for $5 \mathrm{~min}$ at RT. Color change of the mixture was graded from zero (no activity) to five by comparing color intensity with the color chart provided by the manufacturer. Positive enzyme activities were determined to be above three intensity levels of color change.

\section{BA Production of $B$. breve IDCC4401}

Production of BAs by $B$. breve IDCC4401 was investigated following the method described in a previous study [22] with minor modifications. The overnight cultured B. breve IDCC 4401 was centrifuged at $2,300 \times g$ for $5 \mathrm{~min}$ at $4^{\circ} \mathrm{C}$. An aliquot of $0.75 \mathrm{ml}$ of supernatant was mixed with the same volume of $0.1 \mathrm{M} \mathrm{HCl}$ and filtered through a $0.45-\mu \mathrm{m}$ membrane to extract BAs. For derivatization of the BAs, $1 \mathrm{ml}$ of filtered BAs was incubated at $70^{\circ} \mathrm{C}$ for $10 \mathrm{~min}$ prior to addition of $200 \mu \mathrm{l}$ of saturated $\mathrm{NaHCO}_{3}, 20 \mu \mathrm{l}$ of $2 \mathrm{M} \mathrm{NaOH}$, and $0.5 \mathrm{ml}$ of dansyl chloride solution $\left(10 \mathrm{mg} / \mathrm{ml}\right.$ of acetone). The derivatized BAs were mixed with $200 \mu \mathrm{l}$ of proline $\left(100 \mathrm{mg} / \mathrm{ml} \mathrm{of}_{2} \mathrm{O}\right)$ and incubated for $15 \mathrm{~min}$ at RT in the dark. The derivatized BAs were then separated and quantified using HPLC (LC-NETII/ ADC, JASCO Inc., Japan) with an Athena C18 column (4.6 mm × $250 \mathrm{~mm}$, ANPEL Laboratory Technologies Inc., China). Aqueous acetonitrile solution (Sigma-Aldrich Co., USA) was used as a mobile phase and the flow rate was adjusted to $0.8 \mathrm{ml} / \mathrm{min}$. Finally, a peak was detected at $254 \mathrm{~nm}$ using a UV detector (UV-2075 Plus, JASCO Inc., 
Japan). The detected BAs were quantified from calibration curves of BAs including tyramine, histamine, putrescine, 2-phenethylamine, and cadaverine (Sigma-Aldrich Co.).

\section{Proportion of D-/L-Lactate of B. breve IDCC4401}

An overnight culture of $B$. breve IDCC4401 was centrifuged at $2,300 \times g$ for $30 \mathrm{~min}$ at $4^{\circ} \mathrm{C}$ and the supernatant was collected. Following supernatant filtration using a $0.2-\mu \mathrm{m}$ pore size membrane, the filtrate was mixed with the agents in a D-/L-Lactic Acid (D-/L-Lactate) (Rapid) Assay Kit (Megazyme, Ireland). Absorbances of the mixture were measured at $340 \mathrm{~nm}$ and the concentration of D-/L-lactate was calculated according to the manufacturer's protocol.

\section{Results and Discussion}

\section{Whole Genome Sequencing of B. breve IDCC4401}

Whole genome sequencing of $B$. breve IDCC4401 was performed for the identification and confirmation of genes encoding antibiotic resistance, virulence, and mobile genetic elements to ensure safety. The assembled genome consisted of 2,426,499 bp with a GC content of $58.70 \%$ (Fig. 1). The strain was confirmed as B. breve with a similarity of $98.93 \%$ with B. breve DSM20213 based on ANI analysis. Of a total of 2,016 CDSs of B. breve IDCC4401, 1,583 CDSs were annotated as functional genes involved in translation, ribosomal structure, biogenesis, RNA processing, modification, transcription, replication, recombination, repair, cell cycle control, cell division, chromosome partitioning, defense mechanisms, signal transduction mechanisms, and 433 unknown genes (Table 1). Based on CARD and VFDB, genes associated with virulence and antibiotic resistance were not found in B. breve IDCC4401, respectively. Although 33 transposases, as mobile genetic elements, were identified, these mobile elements were not involved in the acquisition and transfer of antibiotic resistance genes due to the absence of virulence and antibiotic resistance genes in B. breve IDCC4401. Therefore, this result confirmed the safety of B. breve IDCC4401 for use as a probiotic based on our thorough genome analysis.

\section{Antibiotic Susceptibility (MICs) of B. breve IDCC4401}

To ensure safety, the phenotypic antibiotic susceptibility of B. breve IDCC4401 was investigated against 9 antibiotics including ampicillin (one representative of a $\beta$-lactam antibiotic), gentamicin, streptomycin, erythromycin, clindamycin, tetracycline, chloramphenicol, vancomycin, and kanamycin, using the E-test method [18]. Determination of resistance and susceptibility against each antibiotic followed the EFSA guidelines. As shown in Table 2, B. breve IDCC4401 was susceptible to ampicillin, gentamicin, streptomycin, erythromycin, clindamycin, tetracycline, and chloramphenicol but resistant to vancomycin. Susceptibility to kanamycin could not be determined because EFSA did not provide a definite cut-off value. However, the MIC value $(256 \mu \mathrm{g} / \mathrm{ml})$ against kanamycin was 2 times lower than that of B. longum BORI $(512 \mu \mathrm{g} / \mathrm{ml})$, and 4 times lower than those of B. longum BB536, B. breve M-16, B. bifidum BGN4, and B. lactis BB-12 $(1,024 \mu \mathrm{g} / \mathrm{ml})$ that were considered as GRAS [16].

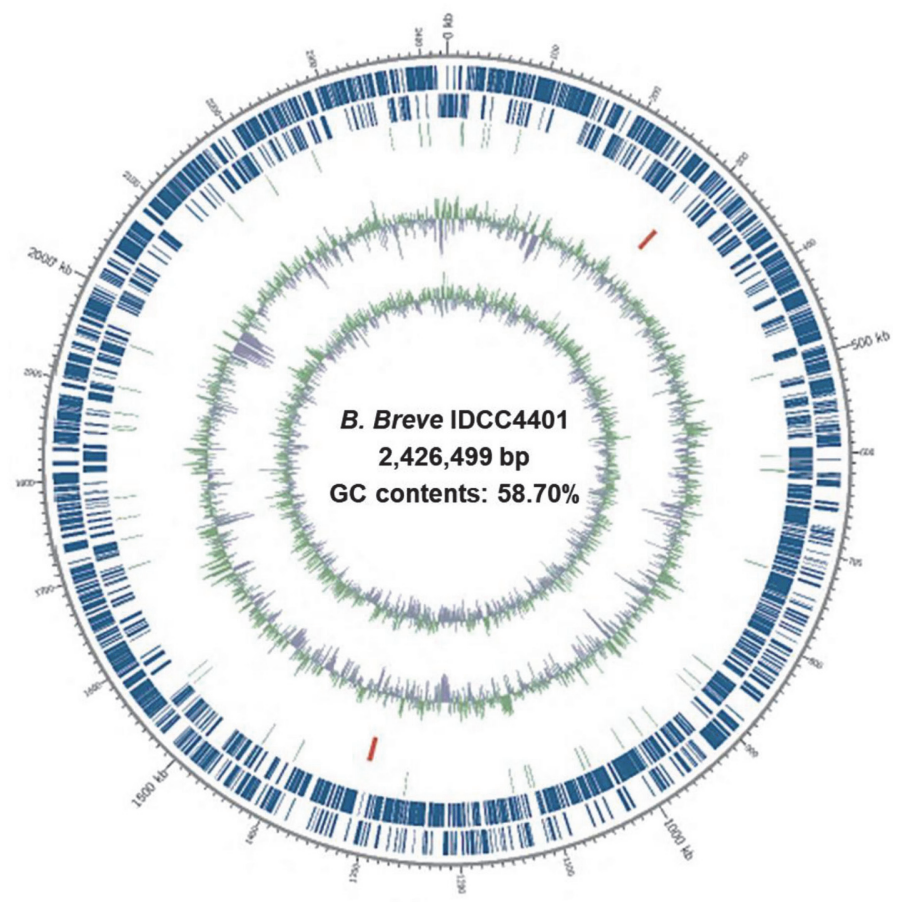

Fig. 1. Genomic map of B. breve IDCC4401. Marked genome characteristics are shown from outside to the center; CDS on forward strand, CDS on reverse strand, tRNA, rRNA, GC content and GC skew. 
Table 1. Annotated functional genes in B. breve IDCC4401.

\begin{tabular}{lcc}
\multicolumn{1}{c}{ Function } & Number of CDS & Ratio (\%) of CDS \\
\hline Translation, ribosomal structure, and biogenesis & 134 & 6.6468 \\
RNA processing and modification & 1 & 0.0496 \\
Transcription & 125 & 6.2004 \\
Replication, recombination, and repair & 191 & 9.4742 \\
Cell cycle control, cell division, chromosome partitioning & 19 & 0.9425 \\
Defense mechanisms & 52 & 2.5794 \\
Signal transduction mechanisms & 53 & 2.6290 \\
Cell wall/membrane/envelope biogenesis & 90 & 4.4643 \\
Intracellular trafficking, secretion, and vesicular transport & 14 & 0.6944 \\
Posttranslational modification, protein turnover, chaperones & 51 & 2.5298 \\
Energy production and conversion & 47 & 2.3313 \\
Carbohydrate transport and metabolism & 231 & 11.4583 \\
Amino acid transport and metabolism & 166 & 8.2341 \\
Nucleotide transport and metabolism & 62 & 3.0754 \\
Coenzyme transport and metabolism & 34 & 1.6865 \\
Lipid transport and metabolism & 28 & 1.3889 \\
Inorganic ion transport and metabolism & 95 & 4.7123 \\
Secondary metabolites biosynthesis, transport and catabolism & 9 & 0.4464 \\
General function prediction only & 181 & 8.9782 \\
Unknown function & 433 & 21.4782
\end{tabular}

Table 2. Minimal inhibitory concentration (MIC) and antibiotic susceptibility of B. breve IDCC4401.

\begin{tabular}{lccc}
\hline & Cut-off value $(\mu \mathrm{g} / \mathrm{ml})$ & MIC $(\mu \mathrm{g} / \mathrm{ml})$ & Assessment \\
\hline Ampicillin & 2 & $0.25-0.5$ & $\mathrm{~S}^{1}$ \\
Vancomycin & 2 & $>512$ & $\mathrm{R}^{2}$ \\
Gentamicin & 64 & 32 & $\mathrm{~S}$ \\
Kanamycin & -3 & 256 & - \\
Streptomycin & 128 & $64-128$ & $\mathrm{~S}$ \\
Erythromycin & 1 & $0.125-0.25$ & $\mathrm{~S}$ \\
Clindamycin & 1 & 0.25 & $\mathrm{~S}$ \\
Tetracycline & 8 & 2 & $\mathrm{~S}$ \\
Chloramphenicol & 4 & 4 & $\mathrm{~S}$ \\
\hline
\end{tabular}

${ }^{1} \mathrm{~S}$, Susceptible

${ }^{2} \mathrm{R}$, Resistant

${ }^{3}$-, Cut-off value is not established in EFSA guidelines.

Charteris et al. (1999) investigated the antibiotic susceptibilities of two B. breve strains (15698 and 15701) isolated from human gastrointestinal tract and found resistances against five $\beta$-lactam antibiotics (penicillin G, amoxycillin, cepharadine, ceftizoxime and cefotaxime) among 44 antibiotics considered as "last resort antibiotics" [23]. In the meantime, B. breve IDCC4401 exhibited susceptibility to ampicillin. In addition, two other B. breve strains (IF2-173 and IF2-174) also isolated from breast-fed infants were susceptible to ampicillin, streptomycin, and chloramphenicol whereas they were resistant to tetracycline, erythromycin, and vancomycin [10]. When compared with previous study [10], B. breve IDCC4401 showed narrow antibiotic resistance only to vancomycin. Although $B$. breve IDCC 4401 showed antibiotic resistance against vancomycin, this result could be related to an intrinsic feature of $B$. breve IDCC4401 as vancomycin resistance is a general feature of most Bifidobacterium spp. as shown in a study by Charteris et al. [23]. Vancomycin resistance is thought to be a result of the presence of $\mathrm{D}$-alanine residues in the cell wall preventing vancomycin binding [24,25].

\section{Enzyme Activity of B. breve IDCC4401}

The enzyme activity of $B$. breve IDCC4401 was investigated against 19 different enzymes using the API ZYM assay, which involved degradation of peptides, phosphomonoesters, lipids, mucopolysaccharides, polysaccharides, chitin, cellulose, starch, and galactan (Table 3) [26]. For example, leucine and cysteine arylamidases are involved in the degradation of peptides whereas $\alpha$-galactosidase, $\beta$-galactosidase, and $\alpha$-glucosidase participate in the degradation of carbohydrates. B. breve IDCC4401 exhibited leucine arylamidase, cystine arylamidase, $\alpha$ galactosidase, $\beta$-galactosidase, and $\alpha$-glucosidase activities among the 19 different enzymes.

Desjardins et al's study demonstrated that four B. breve strains (ATCC 15698, ATCC 15699, ATCC 15700, and ATCC 15701) obtained from the American Type of Culture Collection (ATCC) exhibited the activities of esterase lipase, leucine aminopeptidase, acid phosphatase, phosphoamidase, $\alpha$-galactosidase, $\beta$-galactosidase, $\alpha$-glucosidase, and $\beta$-glucosidase [27]. In addition, $B$. breve ATCC 15699 showed $\beta$-glucuronidase activity. The enzyme activities of $\alpha$-galactosidase, $\beta$-galactosidase and $\alpha$-glucosidase were found to be consistent with those of a previous study [27]. Chevalier et al. (1990) demonstrated that the presence of Bifidobacterium spp. in the feces of the healthy 
Table 3. Enzyme activities of B. breve IDCC4401 determined by API ZYM test.

\begin{tabular}{ll}
\hline \multicolumn{1}{c}{ Enzyme } & Activity $^{*}$ \\
\hline Alkaline phosphatase & - \\
Esterase & - \\
Esterase Lipase & - \\
Lipase & - \\
Acid phosphatase & - \\
Naphthol-AS-BI-phosphohydrolase & + \\
Leucine arylamidase & - \\
Valine arylamidase & + \\
Cystine arylamidase & - \\
Trypsin & - \\
$\alpha$-Chymotrypsin & + \\
$\alpha$-Galactosidase & + \\
$\beta$-Galactosidase & + \\
$\beta$-Glucuronidase & +- \\
$\beta$-Glucosidase & + \\
N-Glucosidase & - -acetyl- $\beta$-glucosaminidase \\
$\alpha$-Mannosidase & - \\
$\alpha$-Fucosidase & -
\end{tabular}

*+, enzyme activity; -, no enzyme activity

infants was accompanied by a-galactosidase and $\alpha$-glucosidase, which are characteristics of Bifidobacterium spp. [28]. Further, evidence proved that $\alpha$-galactosidase and $\beta$-galactosidase enzyme activities were highly produced in B. longum $\mathrm{RD} 47$, suggesting that these enzymes are involved in hydrolyzing galactosides such as lactose [29]. These findings are consistent with our results obtained from this study. Although probiotics can improve digestion and probiotic enzymes can act as natural substances for digestion of food in the human body, some enzyme activities may produce compounds that are to the host [30]. Cole and Fuller (1986) reported that $\beta$ glucosidase may produce aglycones which are linked to the development of colorectal cancer [31]. Moreover, $\beta$ glucuronidase might be linked to carcinogenic compounds for colorectal cancer [32]. Since B. breve IDCC4401 did not show the activity of $\beta$-glucosidase and $\beta$-glucuronidase, it appears to be safe and suitable for use as a probiotic.

\section{BA Production of $B$. breve IDCC4401}

Lactic acid bacteria (LAB) including Bifidobacterium spp. and Lactobacillus spp. can produce BAs during the fermentation process [33]. Previous studies [4,34] reported that tyramine, histamine, putrescine, cadaverine, 2phenethylamine, and spermidine can be frequently detected in fermented foods. The problem is that these BAs, at high concentration, can cause toxicological effects to humans with certain symptoms such as respiratory distress, heart palpitation, hypertension or hypotension, headaches, and allergenic disorders [33]. Verifying the formation of BAs by B. breve IDCC 4401 is required to ensure the safety of $B$. breve IDCC4401. As shown in Table 4, B. breve IDCC4401 did not produce any of the five representative BAs, indicating that it did not produce any harmful BAs.

According to Lorencova et al.'s study [35], five Bifidobacterium strains (Bifidobacterium spp., B. adolescentis, B. lactis, B. bifidum, and B. longum) produced tyramine, cadaverine, putrescine, and spermidine among the eight BAs including the five we tested and three others (tryptamine, speramine, and spermidine). However, Kim et al's study [16] exhibited putrescine formation by B. bifidum BGN4 and B. longum BORI, but not cadaverine, histamine, or tyramine formation. Contradictory to previous studies, Ku et al. [4] demonstrated that B. lactis AD011 did not form any BAs when tested for cadaverine, histamine, putrescine, and tyramine formation, in accordance with our results. Thus, the absence of BAs suggested the potential of B. breve IDCC4401 as a commercial probiotic.

\section{Proportion of D-/L-Lactate of B. breve IDCC4401}

Lactate produced in either $\mathrm{D}$-form or L-form isomers during fermentation by LAB and exhibits distinct biological effects in human [36]. However, Lactobacillus and Bifidobacterium have been known as D-lactate producers [37, 38]. Unlike L-lactate, excess D-lactate produced by LAB cause short bowel syndrome, chronic fatigue and metabolic disorders, especially when jejunoileal bypass surgery is performed [39]. Due to these

Table 4. Biogenic amine production of B. breve IDCC4401.

\begin{tabular}{ccccc}
\hline \multicolumn{4}{c}{ Biogenic amine $(\mu \mathrm{g} / \mathrm{ml})$} \\
\hline Tyramine & Histamine & Putrescine & Cadaverine & 2-Phenethylamine \\
\hline ND. & ND. & ND. & ND. & ND. \\
\hline
\end{tabular}

ND: not detected. 
Table 5. L-/D-lactate production of B. breve IDCC4401.

\begin{tabular}{cccc}
\hline L-lactate $(\mathrm{g} / \mathrm{l})$ & D-lactate $(\mathrm{g} / \mathrm{l})$ & L-form $(\%)$ & D-form $(\%)$ \\
\hline 21.26 & 0.93 & 95.08 & 4.92 \\
\hline
\end{tabular}

The data represent the mean $\pm \mathrm{SD}(n=3)$.

toxicological effects, the proportion of D-/L-lactate of B. breve IDCC4401 needs to be clarified for its safety evaluation. As shown in Table 5, B. breve IDCC4401 produced $95.08 \%$ (21.26 g/l) of L-lactate and 4.92\% (0.93 g/l) of D-lactate.

Since the guidelines provided by FAO/WHO did not provide any clear criterion for the ratio of L-lactate and Dlactate, these results were compared with other studies recognized as GRAS. According to Munoz et al's study [40], B. longum CECT 7210 produced $2.22 \%(0.06 \mathrm{~g} / \mathrm{l})$ of D-lactate, which was lower than the L-lactate $(97.78 \%, 2.64 \mathrm{~g} / \mathrm{l})$. Although the amount and ratio of D-lactate produced by B. breve IDCC4401 was higher than that of $B$. longum CECT 7210, it was lower than that of L-lactate and this pattern was similar to a previous study. In the case of $B$. lactis BB-12 which was certified as GRAS by the FDA, the ratio of L-lactate was more than 95\% [41]. In this aspect, it can be assumed that $B$. breve IDCC4401 poses no safety concern over this property.

In conclusion, since the safety of $B$. breve IDCC4401 isolated from infant feces has not yet been investigated for its commercial usage as a probiotic, we investigated the presence of genes encoding virulence, antibiotic resistance, mobile genetic elements, and deleterious metabolic activities in this strain to ascertain its safety as a probiotic. Although B. breve IDCC4401 was evaluated for safety in this study, further studies are required on functional characteristics such as resistance to gastric acid and bile salts, adherence ability (aggregation properties and cell hydrophobicity), and antimicrobial activity to ensure its safety as a commercial probiotic in the food industry.

\section{Acknowledgments}

This work was supported by Ildong Bioscience Co.

\section{Conflict of Interest}

The authors have no financial conflicts of interest to declare.

\section{References}

1. FAO/WHO. 2001. Report of a joint FAO/WHO expert consultation on evaluation of health and nutritional properties of probiotics in food including powder milk with live lactic acid bacteria. Córdoba, Argentina: Food and Agricultural Organization of the United Nations, World Health Organization.

2. Kechagia M, Basoulis D, Konstantopoulou S, Dimitriadi D, Gyftopoulou K, Skarmoutsou N, et al. 2013. Health benefits of probiotics: a review. ISRN Nutr. 2013: PMC4045285.

3. Lim HJ, Shin HS. 2020. Antimicrobial and immunomodulatory effects of Bifidobacterium strains: a review. J. Microbiol. Biotechnol. 30: 1793-1800.

4. Ku S, Yang S, Lee HH, Choe D, Johnston TV, Ji GE, et al. 2020. Biosafety assessment of Bifidobacterium animalis subsp. lactis AD011 used for human consumption as a probiotic microorganism. Food Control 117: 106985.

5. O'Shea EF, Cotter PD, Stanton C, Ross RP, Hill CJIjofm. 2012. Production of bioactive substances by intestinal bacteria as a basis for explaining probiotic mechanisms: bacteriocins and conjugated linoleic acid. Int. J. Food Microbiol. 152: 189-205.

6. Jin H, Jeong Y, Yoo S-H, Johnston TV, Ku S, Ji GEJMcf. 2019. Isolation and characterization of high exopolysaccharide-producing Weissella confusa VP30 from young children's feces. Microb. Cell Fact. 18: 110.

7. GVR. 2019. Probiotics market size, share and trends analysis report by product (food and beverages, dietary supplements), by ingredient (bacteria, yeast), by end use, by distribution channel, and segment forecasts, 2019-2025. Available from https:// www.grandviewresearch.com/industry-analysis/probiotics-market. Accessed Mar. 17, 2021.

8. Ciorba M. 2012. A gastroenterologist's guide to probiotics. Clin. Gastroenterol. Hepatol. 10: 960-968.

9. Lee J-H, O'Sullivan DJJM, Reviews MB. 2010. Genomic insights into bifidobacteria. Microbiol. Mol. Biol. Rev. 74: 378-416.

10. Zuo F, Yu R, Feng X, Chen L, Zeng Z, Khaskheli GB, et al. 2016. Characterization and in vitro properties of potential probiotic Bifidobacterium strains isolated from breast-fed infant feces. Ann. Microbiol. 66: 1027-1037.

11. Tissier H. 1900. Recherches sur la flore intestinale des nourrissons:(état normal et pathologique). University of Paris.

12. Zinedine A, Faid M. 2007. Isolation and characterization of strains of Bifidobacteria with probiotic proprieties in vitro. WJDFS 2: 28-34.

13. Sgorbati B, Biavati B, Palenzona D. 1995. The genus Bifidobacterium, pp. 279-306. In Wood BJB, Holzapfeel WH (eds.), The genera of lactic acid bacteria, Ed. Springer, Boston, MA, USA.

14. Koirala S, Anal AK. 2021. Probiotics-based foods and beverages as future foods and their overall safety and regulatory claims. Future Foods 3: 100013.

15. FAO/WHO. 2002. Working group report on drafting guidelines for the evaluation of probiotics in food. World Health Organization and Food and Agriculture Organization of the United Nations. Ontario: London. UK.

16. Kim MJ, Ku S, Kim SY, Lee HH, Jin H, Kang S, et al. 2018. Safety evaluations of Bifidobacterium bifidum BGN4 and Bifidobacterium longum BORI. Int. J. Mol. Sci. 19: 1422.

17. EFSA. 2012. Guidance on the assessment of bacterial susceptibility to antimicrobials of human and veterinary importance. EFSA J. 10: $1-10$.

18. FAO/WHO. 2006. Probiotics in food: Health and nutritional properties and guidelines for evaluation. World Health Organization and Food and Agriculture Organization of the United Nations. Rome: Italy.

19. McArthur AG, Waglechner N, Nizam F, Yan A, Azad MA, Baylay AJ, et al. 2013. The comprehensive antibiotic resistance database. Antimicrob. Agents Chemother. 57: 3348-3357.

20. Chen L, Zheng D, Liu B, Yang J, Jin Q. 2016. VFDB 2016: hierarchical and refined dataset for big data analysis--10 years on. Nucleic Acids Res. 44: D694-697. 
21. Arndt D, Grant JR, Marcu A, Sajed T, Pon A, Liang Y, et al. 2016. PHASTER: a better, faster version of the PHAST phage search tool. Nucleic Acids Res. 44: W16-W21.

22. Deepika Priyadarshani WM, Rakshit SK. 2011. Screening selected strains of probiotic lactic acid bacteria for their ability to produce biogenic amines (histamine and tyramine). Int. J. Food Sci. Technol. 46: 2062-2069.

23. Charteris W, Kelly P, Morelli L, Collins J. 1998. Antibiotic susceptibility of potentially probiotic Bifidobacterium isolates from the human gastrointestinal tract. Lett. Appl. Microbiol. 26: 333-337.

24. Álvarez-Cisneros YM, Ponce-Alquicira E. 2018. Antibiotic resistance in lactic acid bacteria, pp. 53-73. In Kumar Y (ed.), Antimicrobial Resistance-A Global Threat, Ed. IntechOpen, London, UK

25. Elisha BG, Courvalin P. 1995. Analysis of genes encoding D-alanine: D-alanine ligase-related enzymes in Leuconostoc mesenteroides and Lactobacillus spp. Gene 152: 79-83.

26. Martinez D, Molina M, Sanchez J, Moscatelli M, Marinari S. 2016. API ZYM assay to evaluate enzyme fingerprinting and microbial functional diversity in relation to soil processes. Biol. Fertil Soils. 52: 77-89.

27. Desjardins M-L, Roy D, Goulet J. 1990. Growth of bifidobacteria and their enzyme profiles. J. Dairy Sci. 73: 299-307.

28. Chevalier P, Roy D, Ward P. 1990. Detection of Bifidobacterium species by enzymatic methods. J. Appl. Bacteriol. 68: 619-624.

29. Han YR, Youn SY, Ji GE, Park MS. 2014. Production of alpha- and beta-galactosidases from Bifidobacterium longum subsp. longum RD47. J. Microbiol. Biotechnol. 24: 675-682.

30. De Preter V, Raemen H, Cloetens L, Houben E, Rutgeerts P, Verbeke K. 2008. Effect of dietary intervention with different pre-and probiotics on intestinal bacterial enzyme activities. Eur. J. Clin. Nutr. 62: 225-231.

31. Cole C, Fuller R. 1987. The effect of dietary fat and yoghurt on colonic bacterial enzymes ( $\beta$-glucosidase and $\beta$-glucuronidase) associated with colon cancer. Food Microbiol. 4: 77-81.

32. Kim DH, Jin YH. 2001. Intestinal bacterial $\beta$-glucuronidase activity of patients with colon cancer. Arch Pharm. Res. 24: 564-567.

33. Barbieri F, Montanari C, Gardini F, Tabanelli G. 2019. Biogenic amine production by lactic acid bacteria: a review. Foods. 8: 17.

34. Ekici K, Omer AK. 2018. Detection of common biogenic amines in fermented sausage produced in Turkey. Data Brief. 20: 13601362.

35. Lorencová E, Buňková L, Matoulková D, Dráb V, Pleva P, Kubáň V, et al. 2012. Production of biogenic amines by lactic acid bacteria and bifidobacteria isolated from dairy products and beer. Int. J. Food Sci. Technol. 47: 2086-2091.

36. Pohanka M. 2020. D-Lactic acid as a metabolite: Toxicology, diagnosis, and detection. Biomed. Res. Int. 2020: 3419034

37. Lee DK, Park JE, Kim MJ, Seo JG, Lee JH, Ha NJ. 2015. Probiotic bacteria, B. longum and L. acidophilus inhibit infection by rotavirus in vitro and decrease the duration of diarrhea in pediatric patients. Clin. Res. Hepatol. Gastroenterol. 39: 237-244.

38. Prasirtsak B, Thitiprasert S, Tolieng V, Assabumrungrat S, Tanasupawat S, Thongchul N. 2019. D-Lactic acid fermentation performance and the enzyme activity of a novel bacterium Terrilactibacillus laevilacticus SK5-6. Ann. Microbiol. 69: 1537-1546.

39. Ewaschuk JB, Naylor JM, Zello GA. 2005. D-lactate in human and ruminant metabolism. J. Nutr. 135: 1619-1625.

40. Muñoz JAM, Chenoll E, Casinos B, Bataller E, Ramón D, Genovés S, et al. 2011. Novel probiotic Bifidobacterium longum subsp. infantis CECT 7210 strain active against rotavirus infections. Appl. Environ. Microbiol. 77: 8775-8783.

41. Hansen C. 2019. Generally recognized as safe (GRAS) determination for the intended use of Bifidobacterium animalis ssp. lactis BB$12^{\circ} \mathrm{FDA}$. 